\title{
(1) Isolated pelvic bone involvement as a presentation of alveolar echinococcosis
}

\author{
Audrey Cambier, Marie-Pierre Hayette, Philippe Léonard, William Kurth, Pierre Lovinfosse, Jean-Baptiste Giot
}

Lancet Infect Dis 2021; 21: 1192 ECHINO-LIEGE (A Cambier MD, M-P Hayette PhD, P Léonard MD, P Lovinfosse MD, J-B Giot MD), Department of Clinical Microbiology (M-P Hayette), Department of General Internal Medicine and Infectious Diseases ( $\mathrm{P}$ Léonard, J-B Giot) Department of Orthopedic Surgery (W Kurth MD), and Department of Nuclear Medicine and Oncological Imaging (P Lovinfosse), CHU de Liège, Liège, Belgium Correspondence to: Dr Audrey Cambier, ECHINO-LIEGE, CHU Liège, Liège 4000, Belgium au.cambier@gmail.com
In 2016, an 85-year-old Belgian woman presented to our hospital with a 2 year history of fistulised collection in the root of the left thigh, associated with weight loss $(20 \mathrm{~kg})$ and sweats, and no evidence of fever. She did not take any immunosuppressive drugs. Having previously worked as a farmer, she had frequent contacts with cattle and dogs. She frequently ate wild berries. She had no history of travelling. Physical examination was unremarkable, except for the thigh collection.

In 2014, she had her first CT imaging of the pelvis at a different hospital due to pain in the left inguinal region, which identified osteolysis of the left pubic rami and acetabulum (figure A, B). ${ }^{18 F}$-fluorodeoxyglucose-PETCT scan only showed an intense heterogeneous hypermetabolism of the osteolytic lesions of the pelvic bone and adjacent soft tissues (figure C). Surgery with cementing of the left acetabulum to fill the osteolytic cavities was done in another hospital. Histology refuted neoplasia but a periodic acid-Schiff-positive laminated layer was consistent with a parasitic disease. Tuberculosis, brucellosis, and syphilis were excluded. After multiple recurrences of fistulae attributed to polymicrobial bacterial infections without definitive resolution, despite several surgeries and longterm antimicrobial regimens, the patient self-referred to our tertiary hospital. Data on antibiotics given before admission to our institution are incomplete; however, the patient had received several treatments including amoxicillinclavulanate, metronidazole, ampicillin, amoxicillin, and ciprofloxacin before presenting to our hospital.

Erythrocyte sedimentation rate was $82 \mathrm{~mm} / \mathrm{h}$, C-reactive protein concentration was $22.8 \mathrm{mg} / \mathrm{L}$, and white cell count was 7780 cells per $\mu \mathrm{L}$ on a blood test. However, serology for
Echinococcus multilocularis was positive, as was a specific E multilocularis PCR test done on new purulent drainage collected by us and on archived tissue harvested in 2014 during cementing surgery of the pelvic bone. We evaluated liver function with an abdominal scan, a PET-CT scan, and a blood test, all of which excluded hepatic involvement.

The diagnosis of isolated bone alveolar echinococcosis without hepatic involvement was retained and we proposed life-long albendazole treatment (400 mg twice a day). After 4 years of treatment, we did not observe any evidence of infection progression and her quality of life is being well maintained within her age category. The patient regained weight and the sweats stopped.

Bone alveolar echinococcosis is a rare presentation, even more so without previous hepatic involvement. However, it is important to inform clinicians of all clinical presentations of this potentially debilitating and fatal disease in the absence of correct diagnosis and appropriate treatment. Alveolar echinococcosis must be excluded in the presence of tumoral lesions in a patient from endemic areas, regardless of the location, especially after neoplasia or other infections are eliminated or when the response to antibiotic treatment is inadequate.

\section{Contributors}

AC collected the data. PL and J-BG did the data analysis. All authors wrote the report. Informed consent was obtained from the patient for publication of this Clinical Picture.

\section{Declaration of interests}

We declare no competing interests.

\section{(C) 2021 Elsevier Ltd. All rights reserved.}
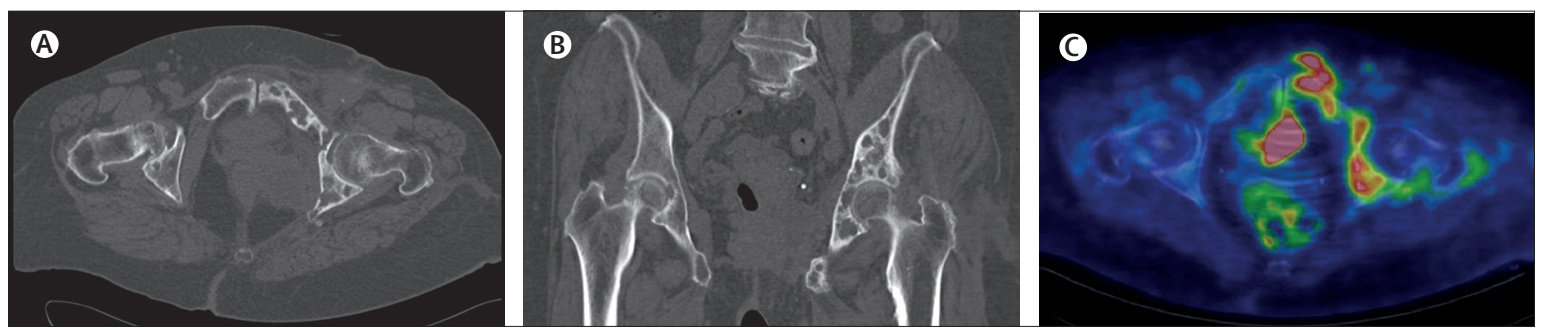

Figure: $\mathrm{CT}$ and PET-CT scan of the pelvis

Transverse (A) and frontal (B) CT scanS of the pelvis showed osteolysis of the left pubic rami and acetabulum. Transverse ${ }^{18} \mathrm{~F}$-fluorodeoxyglucose-PET-CT scan (C) showed an intense heterogeneous hypermetabolism of osteolytic lesion of the pelvic bone and adjacent soft tissues. 